\title{
Time-reversible Born-Oppenheimer molecular dynamics
}

\author{
Anders M. N. Niklasson * \\ Theoretical Division, Los Alamos National Laboratory, Los Alamos, New Mexico 87545 and \\ Applied Materials Physics, Department of Materials Science and Engineering, \\ Royal Institute of Technology, SE-100 44 Stockholm, Sweden \\ C. J. Tymczak, and Matt Challacombe \\ Theoretical Division, Los Alamos National Laboratory, Los Alamos, New Mexico 87545
}

(Dated: July 3, 2018)

\begin{abstract}
We present a time-reversible Born-Oppenheimer molecular dynamics scheme, based on selfconsistent Hartree-Fock or density functional theory, where both the nuclear and the electronic degrees of freedom are propagated in time. We show how a time-reversible adiabatic propagation of the electronic degrees of freedom is possible despite the non-linearity and incompleteness of the selfconsistent field procedure. Time-reversal symmetry excludes a systematic long-term energy drift for a microcanonical ensemble and the number of self-consistency cycles can be kept low (often only 2-4 cycles per nuclear time step) thanks to a good initial guess given by the adiabatic propagation of the electronic degrees of freedom. The time-reversible Born-Oppenheimer molecular dynamics scheme therefore combines a low computational cost with a physically correct time-reversible representation of the dynamics, which preserves a detailed balance between propagation forwards and backwards in time.
\end{abstract}

PACS numbers: 71.15.Pd,31.15.Ew,31.15.Qg,34.10.+x

$A b$ initio molecular dynamics based on Hartree-Fock or density functional theory [1, 2, $3,4,4,5,6,6,8,9]$ has become an important tool for simulations of an increasingly wider range of problems in geology, material science, chemistry, and biology. Ab initio molecular dynamics, where the atomic positions move along classical trajectories, can be categorized in two major groups: Lagrangian Car-Parrinello molecular dynamics and BornOppenheimer molecular dynamics [4, 5, 6, 7, 8, 9, 10, 11, 12. The tremendous success of Car-Parinello molecular dynamics, invented two decades ago [4], is based on its low computational cost combined with the conserved Lagrangian properties of the dynamics. However, unless a Car-Parinello simulation is performed carefully, it may yield results different from Born-Oppenheimer molecular dynamics [5, 6, 13, 14, 15, 16]. In BornOppenheimer molecular dynamics the atomic positions are propagated by forces that are calculated at the selfconsistent electronic ground state for each instantaneous arrangement of the ions. Born-Oppenheimer molecular dynamics is computationally expensive compared to Car-Parinello dynamics because of the requirement to reach a self-consistent field (SCF) solution in each timestep. However, the number of SCF cycles and thus the computational cost can be strongly reduced by using an initial guess for the electronic degrees of freedom $\rho\left(t_{n+1}\right)$ (here represented by the electron density), which is given by an extrapolation from previous time steps [5, 8, 9, 17, 18, 19]. The electronic extrapolation scheme, combined with the SCF procedure, can be seen as an adiabatic propagation of the electronic degrees of freedom,

\footnotetext{
${ }^{*}$ Corresponding author: Email amn@lanl.gov
}

where

$$
\rho\left(t_{n+1}\right)=\operatorname{SCF}\left[\rho\left(t_{n}\right), \rho\left(t_{n-1}\right), \ldots\right] .
$$

Unfortunately, this approach has a fundamental problem. Because of the non-linear and irreversible SCF procedure, which in practice never is complete, the timereversal symmetry of the electronic propagation is broken. This problem does not occur in Lagrangian Car-Parinello molecular dynamics 20], where both the nuclear and the electronic degrees of freedom can be propagated with time-reversible Verlet integrators 21]. The main purpose of this letter is to show how an effective time-reversible propagation of the electronic degrees of freedom is possible in Born-Oppenheimer molecular dynamics, despite an irreversible and approximate $\mathrm{SCF}$ procedure.

Computational schemes using time-reversible integrators give an energy meandering around a constant value that does not drift with time. This stability follows from the time-reversal symmetry, which excludes a steady increase or decrease of the energy (over the Poincare' time) for a periodic motion. Because of the broken timereversal symmetry in the adiabatic propagation of the electron density in Eq. (11), a small but systematic energy drift occurs in the evolution of a microcanonical ensemble, with an accumulating phase space error. The error can be systematically reduced by improving the SCF convergence or be removed completely by using an initial guess in the SCF procedure that is independent of previous time steps [8, [9]. Both these remedies are computationally expensive and using an initial guess that is independent from previous time steps often require a significantly increased number of SCF cycles.

The basic principles for the time-reversible lossless integration of the electronic degrees of freedom is described 

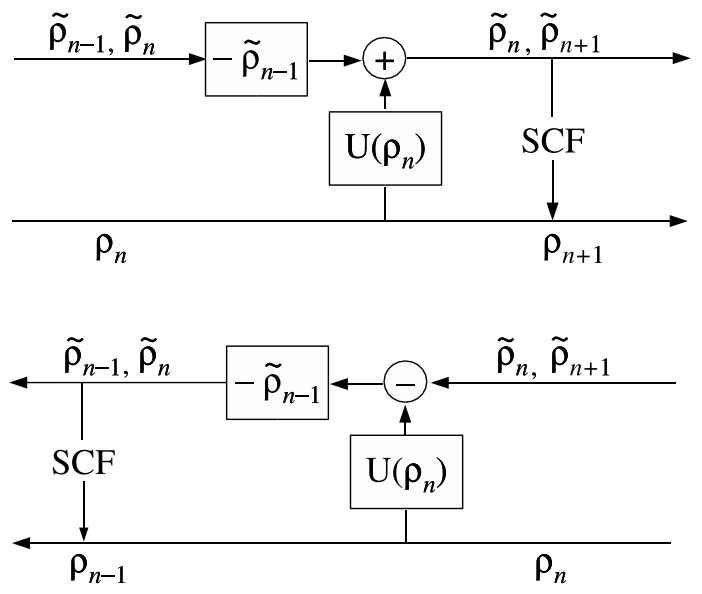

FIG. 1: The principle behind the time-reversible lossless filter process for propagation of the electronic density. In the forward filter process (upper part), $\widetilde{\rho}_{n+1}=-\widetilde{\rho}_{n-1}+U\left(\rho_{n}\right)$ and in the lossless backward reconstruction (lower part), $-\widetilde{\rho}_{n-1}=\widetilde{\rho}_{n+1}-U\left(\rho_{n}\right)$. The dual propagation with the auxiliary density $\tilde{\rho}_{n}$ allows a perfect reconstruction of the self-consistent Born-Oppenheimer density $\rho_{n}$, despite an irreversible, incomplete and approximate $\mathrm{SCF}$ procedure.

here in terms of the propagation of the electron density $\rho(t)$. However, time-reversible Born-Oppenheimer trajectories can be constructed by replacing the density by other parameters governing the electronic degrees of freedom, such as the effective single-particle potential, Hamiltonian, density matrix, or wavefunctions. Our approach is therefore general and applicable to a large number of electronic structure schemes based on selfconsistent Hartree-Fock or density functional theory.

A reversible propagation of the electron density $\rho(t)$ in finite time steps of $\delta t\left(t_{n}=t_{0}+n \delta t\right)$ can be constructed from a lossless filter process analogous to, for example, the lossless wavelet transform used in data compression [22. The principle of the process, which is the key result in this letter, is shown in the upper part of Fig. 1 The scheme is divided into two channels; the upper channel, with an approximate auxiliary density (denoted by a tilde), $\widetilde{\rho}_{n+1} \equiv \widetilde{\rho}\left(t_{n+1}\right)$, is used as an initial guess for the Born-Oppenheimer density, $\rho_{n+1}$, in the lower channel. The Born-Oppenheimer density is given through the non-linear, in practice incomplete, and numerically lossy $\mathrm{SCF}$ procedure,

$$
\rho_{n+1}=\operatorname{SCF}\left[\widetilde{\rho}_{n+1}\right] .
$$

The function $U\left(\rho_{n}\right)$, which is allowed to be numerically approximate and without a unique inverse, is an update filter for the propagation of the auxiliary density,

$$
\widetilde{\rho}_{n+1}=U\left(\rho_{n}\right)-\widetilde{\rho}_{n-1} .
$$

It is easy to see that the filter process is perfectly lossless and reversible by running the process backwards in time with the $\oplus$ sign replaced by a $\ominus$ sign, as shown in the lower part of Fig. 1 The scheme is therefore a bijective map which allows perfect reconstruction of $\rho_{n}$ backwards in time, despite the fact that the SCF procedure by itself is an irreversible lossy transform.

The auxiliary density $\widetilde{\rho}_{n+1}$ in Eq. (3) will be close to the self-consistent Born-Oppenheimer density $\rho_{n+1}$ if the lossless filter process in Fig. 1 approximates the timereversible adiabatic evolution of the density on the BornOppenheimer potential energy surface. This reduces the number of SCF cycles necessary to reach the new selfconsistent density. One way to achieve this is to construct the update filter $U\left(\rho_{n}\right)$ in Eq. (3) from the time-reversible Verlet integrator [21] such that

$$
\widetilde{\rho}_{n+1}=\left[2 \rho_{n}+\delta t^{2} \ddot{\rho}_{n}\right]-\widetilde{\rho}_{n-1} .
$$

This integrator fulfills time-reversal symmetry since it remains the same if we switch the sign of $\delta t$ and thus interchange $\widetilde{\rho}_{n-1}$ with $\widetilde{\rho}_{n+1}$. Note that perfect lossless reconstruction is a necessary, but not a sufficient, condition for time-reversal symmetry. The simplest timereversible approximation of the second order time derivative, $\ddot{\rho}_{n}=\partial^{2} \rho_{n} / \partial t^{2}$, is to set it equal to zero. In this case the lossless propagation of the auxiliary density is

$$
\widetilde{\rho}_{n+1}=2 \rho_{n}-\widetilde{\rho}_{n-1} .
$$

This surprisingly simple difference approximation fulfills time-reversal symmetry, allows a perfect reconstruction backwards in time, and approximates the propagation of the density with a modified linear interpolation from two previous time steps. It also avoids an unstable exponential error growth since the characteristic equation (with $\rho_{n}$ replaced by $\widetilde{\rho}_{n}$ ) has no roots outside the unit circle. If the auxiliary density $\widetilde{\rho}_{n-1}$ in Eq. (5) is replaced by the self-consistent Born-Oppenheimer density $\rho_{n-1}$ the propagation scheme is identical to a linear interpolation. Below we demonstrate how such a modification affects phase-space conservation and the energy drift.

A quite general scheme for constructing efficient timereversible integrators for the approximate adiabatic propagation of the electronic degrees of freedom is given by a least square fit of the ansatz

$$
\widetilde{\rho}(t)=\sum_{m=0}^{M} a_{m} t^{2 m}-\widetilde{\rho}(-t),
$$

to Born-Oppenheimer densities $\rho\left(t_{n}\right)$ at $N$ successive time steps. A similar least square approximation, but without the constraint of time-reversibility, was recently proposed by Pulay and Fogarasi for the extrapolation of the single-particle Hamiltonian in a highly efficient Fock matrix dynamics (FMD) method [8, 9]. Often only 2-3 SCF cycles are necessary in their scheme, but because of the broken time-reversal symmetry a small but systematic energy drift occurs.

By choosing different numbers of fitted values $N$ we can calculate the expansion coefficients $a_{m}$ in Eq. (6) and express them in terms of previous Born-Oppenheimer densities. For example, a least square fit using the ansatz 


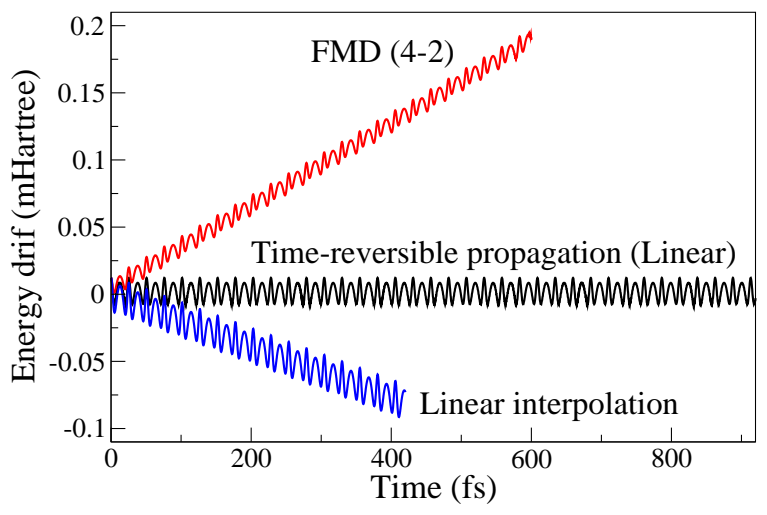

FIG. 2: The fluctuations in the total energy as a function of time for a $\mathrm{F}_{2}$ molecule using Hartree-Fock theory with a Gaussian basis set (RHF/3-21G). The time-reversible propagation based on Eq. (5), with the density replaced by the effective single-particle Hamiltonian, is compared to the energy drift using the corresponding linear interpolation from previous time steps. The (4-2) Fock matrix dynamics (FDM) s

$\mathrm{t}$

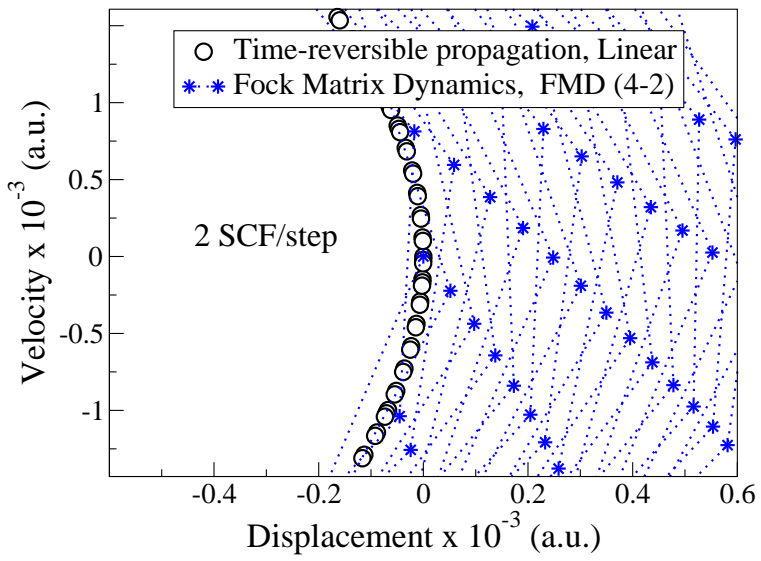

FIG. 3: Detail of the phase space during $500 \mathrm{fs}$ for one of the atoms in the $F_{2}$ molecule (Hartree-Fock theory with a Gaussian basis set RHF/3-21G, $2 \mathrm{SCF} /$ step). The time-reversible propagation preserves the phase space whereas the non-timereversible dynamics has a small but noticeable drift.

in Eq. (6), with $M=1$ for 6 previous densities, leads to the time-reversible approximation

$\widetilde{\rho}_{n+1}=\frac{1}{13}\left[30\left(\rho_{n}+\rho_{n-4}\right)-3\left(\rho_{n-1}+\rho_{n-3}\right)-28 \rho_{n-2}\right]-\widetilde{\rho}_{n-5}$.

Note that the least square ansatz in Eq. (6) also can be extended to negative time exponents that are even.

To demonstrate the time-reversible lossless BornOppenheimer molecular dynamics we have implemented the scheme in MondoSCF [23], a suite of programs using Gaussian basis sets for electronic structure calculations based on self-consistent Hartree-Fock or density functional theory. The density in the time-reversible propagation in Eqs. (5) and (6) has been replaced by the ef- fective single-particle Hamiltonian. The number of SCF cycles has been measured in the number of constructions of Hamiltonians, which is the most time consuming step.

Figure 2 2 shows the total energy as a function of time for an $F_{2}$ molecule. The modification of the time-reversible linear propagation in Eq. (5) to a linear interpolation leads to a systematic drift in the energy. Using more SCF cycles per time step reduces the energy drift, but it never really disappears. As a comparison we also show the (4-2) Fock matrix dynamics (FMD) scheme by $\mathrm{Pu}-$ lay and Fogarasi $[8$, based on a second order polynomial least square fit using four previous data points. This scheme gives, in principle, a more accurate extrapolation, which is noticed in a smaller amplitude of the energy oscillations. However, because of the broken time-reversal symmetry there is a systematic drift in the energy. For the time-reversible linear integrator in Eq. (5), using only 2 SCF iterations per time step, any energy drift was less than $10^{-8}$ Hartree/ps. The phase space is also conserved with the time-reversible integration, as illustrated in Fig. (3). Thus, even with an incomplete SCF convergence time-reversibility is correctly preserved.

Though unproven, all time-reversible propagators we have found so far have characteristic equations with all their roots on the unit circle. Because of the perfect lossless reconstruction, any error that occurs in the calculations will propagate throughout the simulation. This leads to a random noise that increases with time. Because of this noise the auxiliary density $\widetilde{\rho}_{n}$ slowly moves away from the self-consistent solution. This means that it is not possible to simply take long time steps using only $1 \mathrm{SCF}$ cycle per time step. An increased number of SCF cycles (or shorter time steps) is in general necessary to reach a sufficiently accurate Born-Oppenheimer density for longer simulation times. Figure 4 shows the fluctuations in the total energy for a $\mathrm{C}_{2} \mathrm{~F}_{4}$ molecule during $1 \mathrm{ps}$ of simulation time at a temperature $T \approx 500 \mathrm{~K}$. The extrapolation scheme in Eq. (7) was used and 3 SCF cycles per time step was applied using Pulay's direct inversion in the iterative subspace (DIIS) algorithm to accelerate the convergence [24, 25].

The ability of a perfect reconstruction of the dynamical data backwards in time is kept also using approximate arithmetics, thanks to the lossless bijective filter process illustrated in Fig. [1 The lossless property is therefore kept also when small elements below some drop tolerance are set to zero in the SCF optimization of $\rho_{n}$ and in the update function $U\left(\rho_{n}\right)$. This is potentially important in the study of very large systems using, for example, linear scaling electronic structure methods [26] that in general require a lower numerical accuracy compared to conventional schemes.

The main result in this letter is that we have shown that an effective time-reversible propagation of the electronic degrees of freedom is possible in self-consistent Born-Oppenheimer molecular dynamics, despite the irreversible, non-linear, and in practice always approximate $\mathrm{SCF}$ procedure. This ability may also open the way for 


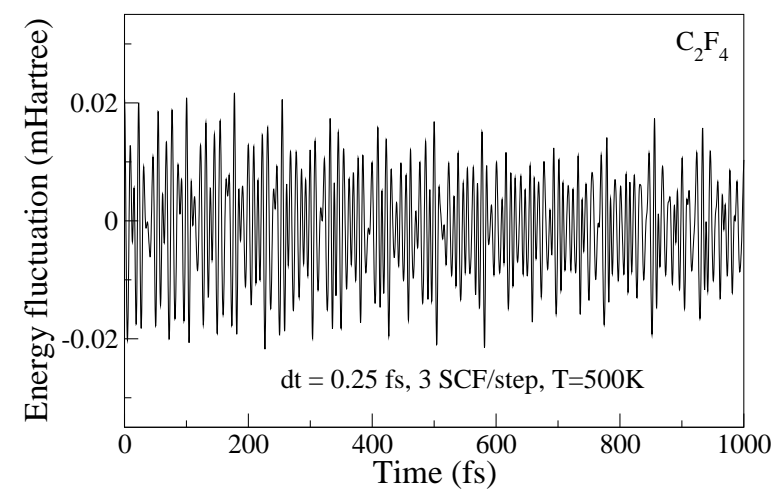

FIG. 4: The energy fluctuations around the average energy as a function of time for a $\mathrm{C}_{2} \mathrm{~F}_{4}$ molecule at a temperature $T \approx 500 \mathrm{~K}$ (Hartree-Fock theory with a Gaussian basis set RHF/3-21G). Three SCF cycles per time step of length $\delta t=$ 0.25 fs were used.

the development of higher order symplecitc integrators
[27, 28, 29, 30, 31] in ab initio molecular dynamics,

In summary, we have presented a scheme for timereversible Born-Oppenheimer molecular dynamics that combines a low computational cost with a physically correct time-reversible representation of the dynamics. The principle is based on a lossless filter integration of the electronic degrees of freedom, where a dual propagation with an auxiliary density allows a perfect reconstruction backwards in time of the self-consistent Born-Oppenheimer density. Time-reversal symmetry removes a systematic long term energy drift for a microcanonical ensemble. This energy stabilization, combined with the adiabatic propagation of an approximate BornOppenheimer electron density, strongly reduces the number of necessary self-consistency cycles, while maintaining an accurate physical description.

This work was performed under the auspices of the US Department of Energy, Office of Science and LANL LDRD/ER program. A.N. acknowledge support form the Swedish Research Council (VR).
[1] C. C. J. Roothaan, Rev. Mod. Phys. 23, 69 (1951).

[2] P. Hohenberg and W. Kohn, Phys. Rev. 136, B:864 (1964).

[3] W. Kohn and L. J. Sham, Phys. Rev. 140, 1133 (1965).

[4] R. Car and M. Parrinello, Phys. Rev. Lett. 55, 2471 (1985).

[5] M. C. Payne, M. P. Teter, D. C. Allan, T. A. Arias, and J. D. Joannopoulos, Rev. Mod. Phys. 64, 1045 (1992).

[6] D. Marx and J. Hutter, Modern Methods and Algorithms of Quantum Chemistry (ed. J. Grotendorst, John von Neumann Institute for Computing, Jülich, Germany, 2000), 2nd ed.

[7] H. B. Schlegel, J. M. Millam, S. S. Iyengar, G. A. Voth, A. D. Daniels, G. Scusseria, and M. J. Frisch, J. Chem. Phys. 114, 9758 (2001).

[8] P. Pulay and G. Fogarasi, Chem. Phys. Lett. 386, 272 (2004).

[9] J. Herbert and M. Head-Gordon, Phys. Chem. Chem. Phys. 7, 3269 (2005).

[10] C. Leforestier, J. Chem. Phys. 68, 4406 (1978).

[11] R. N. Barnett, U. Landman, A. Nitzan, and G. Rajagopal, J. Chem. Phys. 94, 608 (1991).

[12] R. M. Wentzcovitch and J. L. Martins, Solid. State. Commun. 78, 831 (1991).

[13] P. Tangney and S. Scandolo, J. Chem. Phys. 116, 14 (2002).

[14] J. C. Grossman, E. Schwegler, E. W. Draeger, F. Gygi, and G. Galli, J. Chem. Phys. 120, 300 (2004).

[15] E. Schwegler, J. C. Grossman, F. Gygi, and G. Galli, J. Chem. Phys. 121, 5400 (2004).
[16] D. A. Gibson, I. V. Ionova, and E. Carter, Chem. Phys. Lett. 240, 261 (1995).

[17] T. Arias, M. Payne, and J. Joannopoulos, Phys. Rev. Lett. 69, 1077 (1992).

[18] J. Millan, V. Bakken, W. Chen, L. Hase, and H. B. Schlegel, J. Chem. Phys. 111, 3800 (1999).

[19] C. Raynaud, L. Maron, J.-P. Daudey, and F. Jolibois, Phys. Chem. Phys. 6, 4226 (2004).

[20] P. Tangney, J. Chem. Phys. 124, 44111 (2006).

[21] L. Verlet, Phys. Rev. 159, 98 (1967).

[22] A. R. Calderbank, I. Daubechies, W. Sweldens, and B. L. Yeo, Appl. Comput. Harmon. Anal. 5, 332 (1998).

[23] M. Challacombe, E. Schwegler, C. J. Tymczak, C. K. Gan, K. Nemeth, V. Weber, A. M. N. Niklasson, and G. Henkelman, MondoSCF v1.0 $\alpha 9$ (2001), Los Alamos National Laboratory (LA-CC 01-2), Copyright University of California., URL http://www.t12.lanl.gov/home/mchalla/

[24] P. Pulay, Chem. Phys. Let. 73, 393 (1980).

[25] P. Pulay, J. Comput. Chem. 3, 556 (1982).

[26] S. Goedecker, Rev. Mod. Phys. 71, 1085 (1999).

[27] R. Ruth, IEEE Trans. Nucl. Sci. 30, 2669 (1983).

[28] E. Forest and R. Ruth, Physica D 43, 105 (1990).

[29] B. J. Leimkuhler and R. D. Skeel, J. Comput. Phys. 112, 117 (1994).

[30] D. I. Okunbor, J. Comput. Phys. 120, 375 (1995).

[31] G.J.Martyna and M. Tuckerman, J. Chem. Phys. 102, 8071 (1995). 